\title{
Article \\ Hypoxia Inducible Factor 1A Supports a Pro-Fibrotic Phenotype Loop in Idiopathic Pulmonary Fibrosis
}

\author{
Gali Epstein Shochet ${ }^{1,2, *}$, Becky Bardenstein-Wald ${ }^{2}$, Mary McElroy ${ }^{3}$, Andrew Kukuy ${ }^{4}$, Mark Surber ${ }^{5} \mathbb{D}^{\text {, }}$ \\ Evgeny Edelstein ${ }^{6}$, Barak Pertzov ${ }^{2,7}$ (D), Mordechai Reuven Kramer ${ }^{2,7}$ and David Shitrit ${ }^{1,2}$
}

1 Pulmonary Department, Meir Medical Center, Kfar Saba 4428164, Israel; davids3@clalit.org.il

2 Sackler Faculty of Medicine, Tel Aviv University, Tel Aviv 69978, Israel; bekibarden@gmail.com (B.B.-W.); pertzovb@gmail.com (B.P.); Kremerm@clalit.org.il (M.R.K.)

3 Respiratory Pharmacology, Charles River Laboratories, Edinburgh EH33 2NE, UK; Mary.McElroy@crl.com

4 The Leviev Heart Institute, Sheba Medical Center, Ramat Gan 5262000, Israel; dr.a.kukuy@gmail.com

5 Avalyn Pharma, 701 Pike Street suite 1500, Seattle, WA 98101, USA; msurber@avalynpharma.com

6 Pathology Department, Meir Medical Center, Kfar Saba 4428164, Israel; edelshte@clalit.org.il

7 Pulmonary Division, Rabin Medical Center, Petach Tikva 4941492, Israel

* Correspondence: gali.epstein@clalit.org.il; Tel.: +972-97-472-218

\section{check for}

updates

Citation: Epstein Shochet, G.; Bardenstein-Wald, B.; McElroy, M.; Kukuy, A.; Surber, M.; Edelstein, E.; Pertzov, B.; Kramer, M.R.; Shitrit, D Hypoxia Inducible Factor 1A Supports a Pro-Fibrotic Phenotype Loop in Idiopathic Pulmonary Fibrosis. Int. J. Mol. Sci. 2021, 22, 3331. https://doi.org/10.3390/ ijms22073331

Academic Editor: Frank Zaucke

Received: 3 March 2021

Accepted: 22 March 2021

Published: 24 March 2021

Publisher's Note: MDPI stays neutral with regard to jurisdictional claims in published maps and institutional affiliations.

Copyright: (c) 2021 by the authors. Licensee MDPI, Basel, Switzerland. This article is an open access article distributed under the terms and conditions of the Creative Commons Attribution (CC BY) license (https:// creativecommons.org/licenses/by/ $4.0 /)$.
Abstract: Idiopathic pulmonary fibrosis (IPF) is a progressive lung disease with poor prognosis. The IPF-conditioned matrix (IPF-CM) system enables the study of matrix-fibroblast interplay. While effective at slowing fibrosis, nintedanib has limitations and the mechanism is not fully elucidated. In the current work, we explored the underlying signaling pathways and characterized nintedanib involvement in the IPF-CM fibrotic process. Results were validated using IPF patient samples and bleomycin-treated animals with/without oral and inhaled nintedanib. IPF-derived primary human lung fibroblasts (HLFs) were cultured on Matrigel and then cleared using $\mathrm{NH}_{4} \mathrm{OH}$, creating the IPFCM. Normal HLF-CM served as control. RNA-sequencing, PCR and western-blots were performed. HIF1 $\alpha$ targets were evaluated by immunohistochemistry in bleomycin-treated rats with/without nintedanib and in patient samples with IPF. HLFs cultured on IPF-CM showed over-expression of 'HIF1 $\alpha$ signaling pathway' (KEGG, $p<0.0001$ ), with emphasis on SERPINE1 (PAI-1), VEGFA and TIMP1. IPF patient samples showed high HIF1 $\alpha$ staining, especially in established fibrous tissue. PAI-1 was overexpressed, mainly in alveolar macrophages. Nintedanib completely reduced HIF1 $\alpha$ upregulation in the IPF-CM and rat-bleomycin models. IPF-HLFs alter the extracellular matrix, thus creating a matrix that further propagates an IPF-like phenotype in normal HLFs. This pro-fibrotic loop includes the HIF1 $\alpha$ pathway, which can be blocked by nintedanib.

Keywords: IPF; HIF1A; PAI-1; extracellular matrix; bleomycin; nintedanib; in-vivo; signaling; fibrosis

\section{Introduction}

Idiopathic pulmonary fibrosis (IPF) is a progressive and irreversible lung disease [1]. Fibrosis, in response to tissue damage or persistent inflammation, is a pathological hallmark of many chronic diseases [2,3]. Myofibroblasts accumulate in fibrotic lesions and secrete excessive extracellular matrix (ECM) proteins, resulting in distortion of pulmonary structure and scar tissue formation [4]. In addition, they recruit inflammatory cells by secreting various pro-inflammatory and pro-angiogenic cytokines, further aggravating the inflammatory response [5].

Mechanisms of fibrosis progression can be self-sustaining once established, without the need for ongoing inflammatory signaling. As reviewed by Herrera et al. [6], in the absence of exogenous cytokines, IPF-ECM alone can induce normal lung fibroblasts to turn into activated myofibroblasts. Once formed, IPF-ECM sets up a pro-fibrotic feedback loop that is capable of sustaining progressive fibrosis. In support of this hypothesis, our group developed an in-vitro IPF conditioned matrix (IPF-CM) system, which utilizes primary 
human lung fibroblasts derived from patients with IPF (IPF-HLFs). Normal HLFs (N-HLFs) that are added to the established IPF-CM, presented with increased migration and elevated pro-fibrotic markers. This platform allows us to investigate drug function with regard to fibroblast-ECM interaction [7].

To date, nintedanib and pirfenidone are the only approved treatments for IPF, yet they only slow disease progression. Nintedanib is known to inhibit the receptor tyrosine kinases of platelet-derived growth factor (PDGF), fibroblast growth factor (FGF), and vascular endothelial growth factor (VEGF) [8,9]. However, other non-receptor tyrosine kinases and additional targets were suggested to play a role in exerting its antifibrotic effects. For example, nintedanib was shown to mediate the inhibition of TGF- $\beta 1$-induced myofibroblast differentiation via SMAD3 and MAPK inhibition [10] TGF- $\beta 1$, which is the most potent inducer of fibrogenesis, inhibits PHD2 expression and induces HIF1A stabilization in fibroblasts [11], suggesting that the HIF signaling pathway can also be activated without prominent hypoxic conditions.

The relatively high incidence of systemic side effects warrants the development of more efficient, preferably localized, treatments. For this aim, an inhaled version of nintedanib was recently described. Using two animal models $[12,13]$, it was shown that oral-equivalent to superior effects could be obtained administering substantially reduced inhaled doses, with less adverse effects on general health. Moreover, the activity of inhaled pharmacokinetics were further supported using the in-vitro IPF-CM system wherein only short-duration nintedanib exposure was required to achieve similar effects [13].

In the current work, we used RNA-sequence analysis from the IPF-CM model to explore the underlying signaling pathways and characterized nintedanibs' involvement in this process. Results were validated using IPF patient samples and bleomycin-treated animals.

\section{Results}

\subsection{The HIF1A Pathway Is Over-Expressed in N-HLFs Exposed to IPF-CM}

$\mathrm{N}-\mathrm{HLFs}$ cultured for $24 \mathrm{~h}$ on IPF or control N-CM $(n=5)$ were subjected to RNA-seq analysis. A total of over 20,000 genes were discovered, with 234 significantly upregulated targets. Wong et al. recently published a combined list of "Known Pulmonary Fibrosis Genes", which consists of human genes associated with pulmonary fibrosis from several datasets [14]. We compared the 234 upregulated genes to their list and found $25.6 \%$ (60 genes) were related to pulmonary fibrosis (Figure 1A). Analysis of these 234 genes showed significant enrichment for "response to hypoxia" (GO:0001666) and 'response to decreased oxygen levels' (GO:0036293) $(p<0.0001$, FDR $=0.003)$ using WebGestalt [15] (Figure 1B). Additionally, David [16] analysis showed the "cellular response to hypoxia" (GO:0071456) as the most significant term $\left(p=8.3 \times 10^{-5}\right)$. KEGG pathway analysis showed that the "HIF1 $\alpha$ signaling pathway" was the most significant (FDR $=0.000011174$, Enrichment ratio $=8.8089$ ). The upregulated genes related to the HIF1 $\alpha$ pathway are listed in Table 1. This list included several target genes that are implicated in both angiogenesis and vascular endothelial growth factor (VEGF) pathways (FLT1, VEGFA, SERPINE1 and TIMP1). These targets were previously implicated in fibrosis [17-19], presented with a relatively high number of reads, and were thus validated by qPCR $(p<0.05$, Figure $1 C)$. Although GAPDH levels were elevated in the analysis, it was previously shown not to be significantly altered by western blot [7]. For validation purposes, additional house-keeping genes (HPRT1 and beta-actin $(A C T B)$ ) were added, based on the sequencing results that showed no change following IPF-CM exposure (fold change (FC) $0.98, p=0.95$ and 1.02, $p=0.69$, respectively). In addition, HIF1 $\alpha$ was also significantly elevated following exposure to the IPF-CM at the mRNA and the protein levels ( $p<0.05$, Figure 1D,E). Additionally, protein levels of plasminogen activator inhibitor-1 (PAI-1, encoded by SERPINE1) were elevated (Figure 1E). 


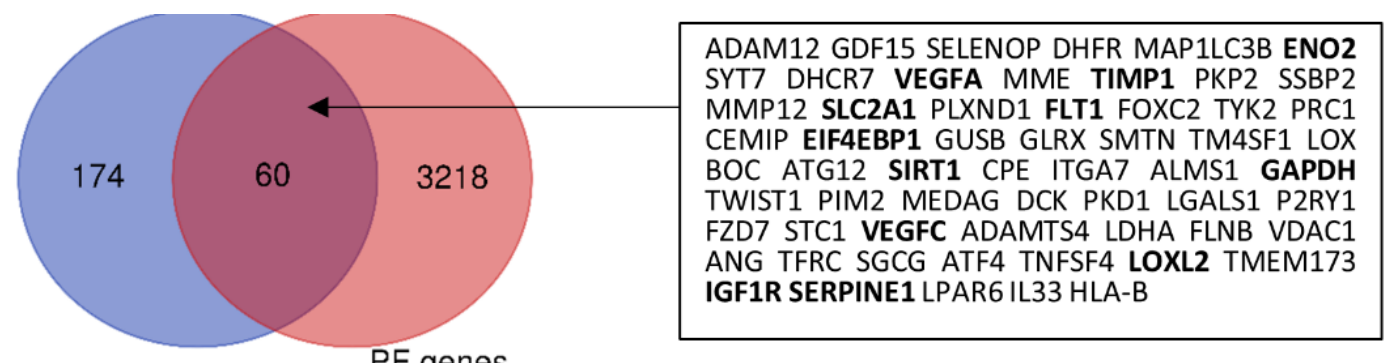

PF genes

B

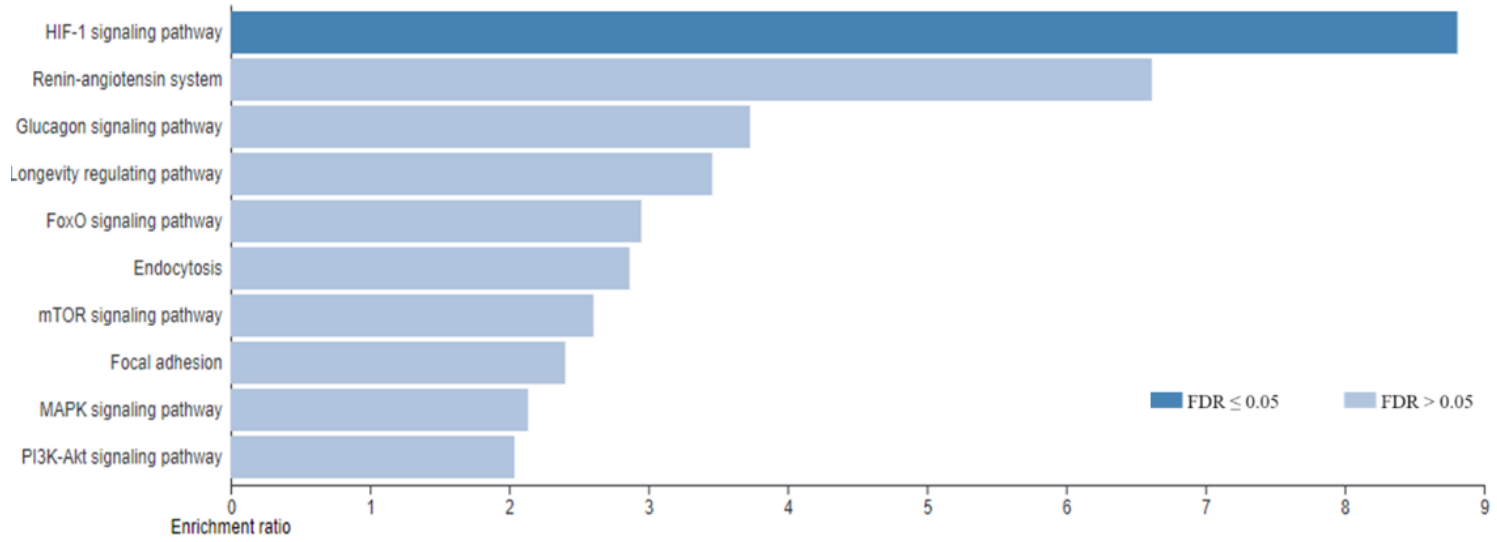

C

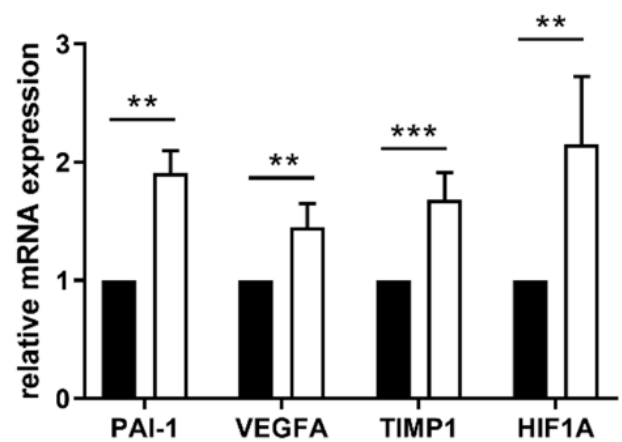

- $\mathrm{N}-\mathrm{CM}$

ㅁ IPF-CM

D

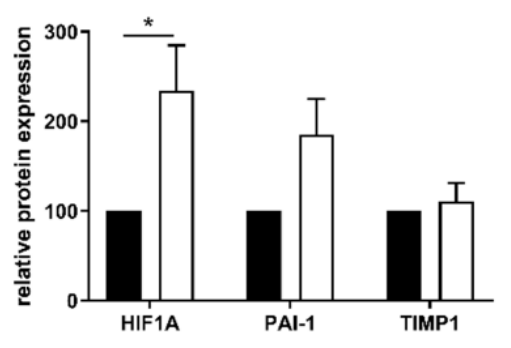

E

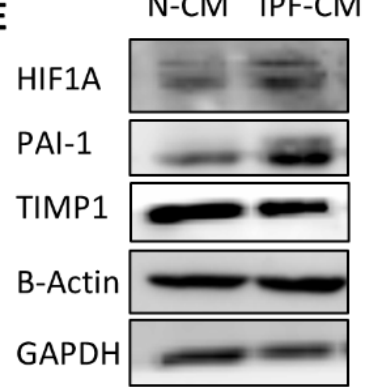

Figure 1. The HIF1 $\alpha$ pathway is over-expressed in N-HLFs exposed to IPF-CM. Normal human lung fibroblasts (N-HLF) were cultured on N/IPF-CM for $24 \mathrm{~h}$. Following culture, RNA was extracted for RNA-sequencing analysis. (A) Venn diagram of the genes elevated in our analysis vs. a list of pulmonary fibrosis (PF) genes. Shared genes are listed in the box. Genes related to the HIF1 $\alpha$ pathway are highlighted in bold. (B) Pathway enrichment analysis (KEGG) using Webgestalt for the upregulated genes. SERPINE1 (PAI-1) $(n=9), V E G F A(n=11)$, TIMP1 $(n=12)$ and HIF1A $(n=8)$ mRNA levels were measured by qPCR $(\mathbf{C})$. The effect of the IPF-CM on N-HLF HIF1 $\alpha(n=12)$, PAI-1 $(n=12)$ and TIMP1 $(n=4)$ protein levels $(24 \mathrm{~h})$ were analyzed by western blotting $(\mathbf{D}, \mathbf{E}){ }^{*} p<0.05,{ }^{* *} p<0.01$, and ${ }^{* * *} p<0.001$. 
Table 1. Genes upregulated in N-HLFs following culture on IPF-CM related to HIF1 $\alpha$ signaling pathway (KEGG).

\begin{tabular}{|c|c|c|c|c|}
\hline Gene Symbol & Gene Name & FC & $\mathbf{p V}$ & \# Reads \\
\hline SLC2A1 & $\begin{array}{l}\text { solute carrier family } 2 \\
\text { member } 1\end{array}$ & 1.456 & 0.00034 & $50-200$ \\
\hline $\mathrm{ENO} 2$ & Enolase 2 & 1.362 & $6.01 \mathrm{E}-06$ & $300-800$ \\
\hline IGF1R & $\begin{array}{l}\text { Insulin-like growth factor } 1 \\
\text { receptor }\end{array}$ & 1.360 & 0.10716 & $30-80$ \\
\hline EIF4EBP1 & $\begin{array}{c}\text { Eukaryotic translation } \\
\text { initiation factor 4E-binding } \\
\text { protein } 1\end{array}$ & 1.355 & 0.14223 & $20-40$ \\
\hline TFRC & Transferrin receptor & 1.13 & 0.03398 & $570-800$ \\
\hline VEGFA & $\begin{array}{l}\text { vascular endothelial growth } \\
\text { factor A }\end{array}$ & 1.281 & 0.00117 & $1440-3400$ \\
\hline GAPDH & $\begin{array}{c}\text { glyceraldehyde-3-phosphate } \\
\text { dehydrogenase }\end{array}$ & 1.219 & 0.00016 & $9-13 \mathrm{~K}$ \\
\hline SERPINE1 & $\begin{array}{l}\text { serpin family E member } 1 \\
\text { (PAI-1) }\end{array}$ & 1.160 & 0.02021 & $2000-4200$ \\
\hline TIMP1 & $\begin{array}{l}\text { Tissue metallopeptidase } \\
\text { inhibitor } 1\end{array}$ & 1.133 & 0.00372 & $12-25 \mathrm{~K}$ \\
\hline FLT1 & Fms related tyrosine kinase 1 & 2.27 & 0.02767 & $3-12$ \\
\hline
\end{tabular}

\subsection{HIF1A-Related Signaling Is Upregulated in Tissue Samples from Patients with IPF}

Analysis of IPF and normal tissue samples $(n=20)$ showed that IPF tissues expressed significantly higher HIF1A, TIMP1, SERPINE1 and VEGFA mRNA levels $(p<0.05$, Figure 2A). IHC staining of paraffin tissue samples from patients with IPF and normal tissue samples revealed that HIF1 $\alpha$ is abundant in the IPF tissue. Further analysis showed that HIF1 $\alpha$ is mostly expressed in alveolar macrophages (AM), basal bronchial epithelial cells and stromal cells within the fibrotic areas. Interestingly, HIF1 $\alpha$ expression was significantly weaker within fibroblastic foci, even in comparison to non-fibrotic areas of the IPF sample $(p<0.001$, Figure 2B,E). Similarly, PAI- 1 was mostly found within AMs and basal bronchial epithelial cells, also showing a similar pattern of lower staining in fibroblastic foci (Figure 2C,E). TIMP1 staining was relatively weak and mostly stromal (Figure 2D,E). 
A
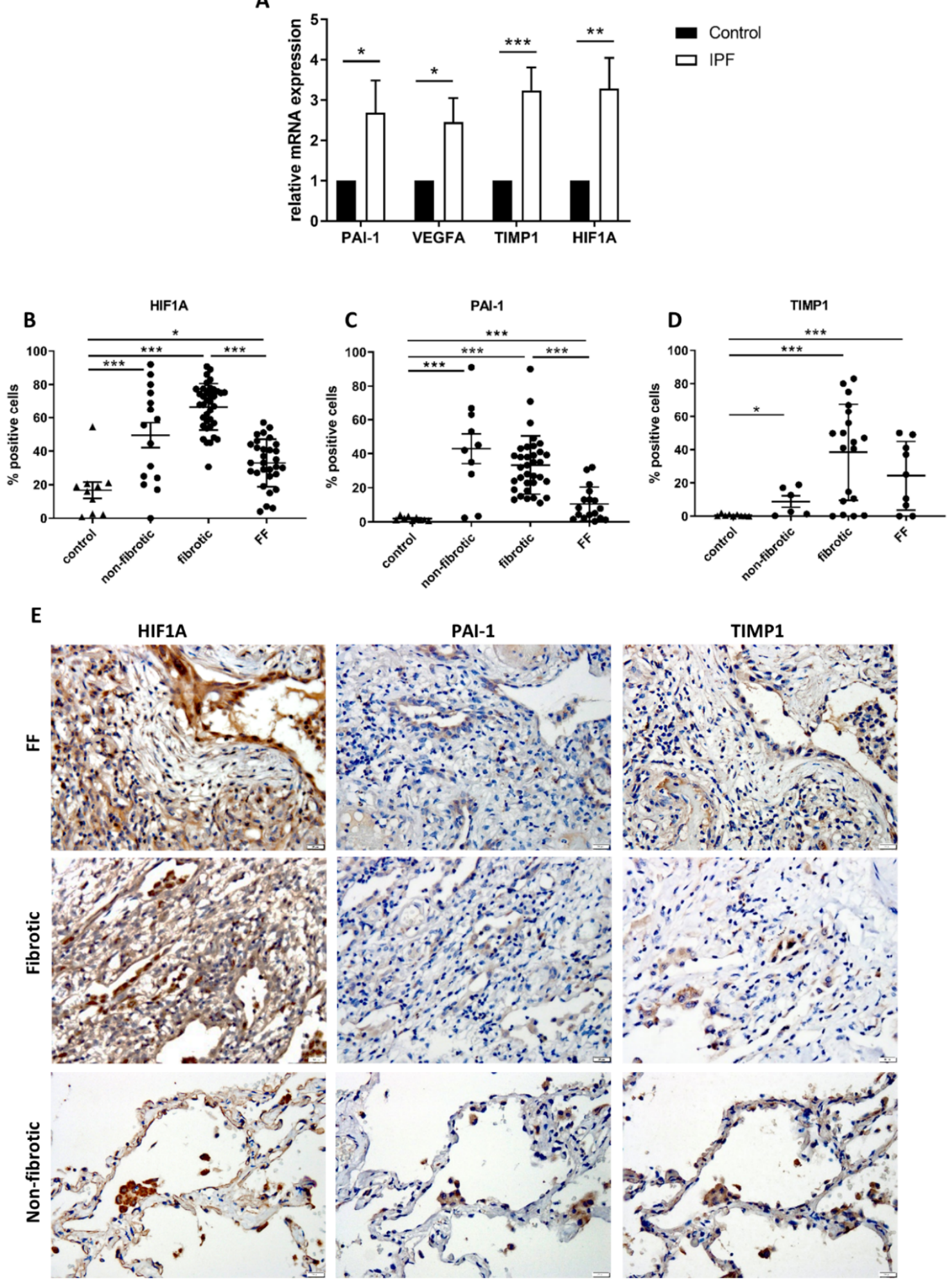

Figure 2. The HIF1 $\alpha$ pathway is overexpressed in IPF patient samples. RNA was extracted from lung tissue lysates derived from patients with IPF or control donors (control). SERPINE1 (PAI-1) $(n=20)$, VEGFA $(n=10)$, TIMP1 $(n=30)$ and HIF1A $(n=30)$ mRNA were measured by qPCR $(\mathbf{A})$. Formalin fixed paraffin embedded sections were stained for HIF1 $\alpha(n \geq 10)$, PAI-1 $(n \geq 10)$, TIMP1 $(n \geq 6)$ by IHC and counter stained by hematoxylin (B-D). Control tissues and Fibroblastic foci (FF), established fibrosis (fibrotic) and non-fibrotic areas of the IPF lung sections were annotated by a registered senior pathologist and quantified. (E) Representative IHC for figures B-D (magnification X200). * One-way ANOVA $p<0.0001$, with the main effect of IPF versus control. Bars represent $t$-tests ${ }^{*} p<0.05$, ${ }^{* *} p<0.01$, and ${ }^{* * *} p<0.001$. 


\subsection{Nintedanib Prevents the Elevation of HIF1 $\alpha$-Related Targets}

Previously, we showed equivalent prevention of IPF-CM effects between long term ( $24 \mathrm{~h}$; oral pharmacokinetic mimic) and short term (60 min; inhalation pharmacokinetic mimic) nintedanib exposure, including inhibition of $\alpha$ SMA and COL1A [13] that were increased as a result of IPF-CM exposure [7]. Here, we tested whether N-HLFs exposed to nintedanib presented with reduced HIF1 $\alpha$ targets. To mimic inhalation, cells were exposed to nintedanib (1-10 nM) for $60 \mathrm{~min}$ prior to IPF-CM challenge. Results indicated that short-duration nintedanib exposure reduced TIMP1, SERPINE1 (PAI-1) and VEGFA mRNA levels $(p<0.05$, Figure 3A-C). In addition, mRNA and protein levels of HIF1 $\alpha$ were reduced $(p<0.05$, Figure 3D,E). Further supporting inhalation as a means to deliver effective nintedanib and correlating to changes in aggregate size and cell count, these results show that short-duration exposure is as effective as continuous exposure in this human derived in-vitro IPF model.
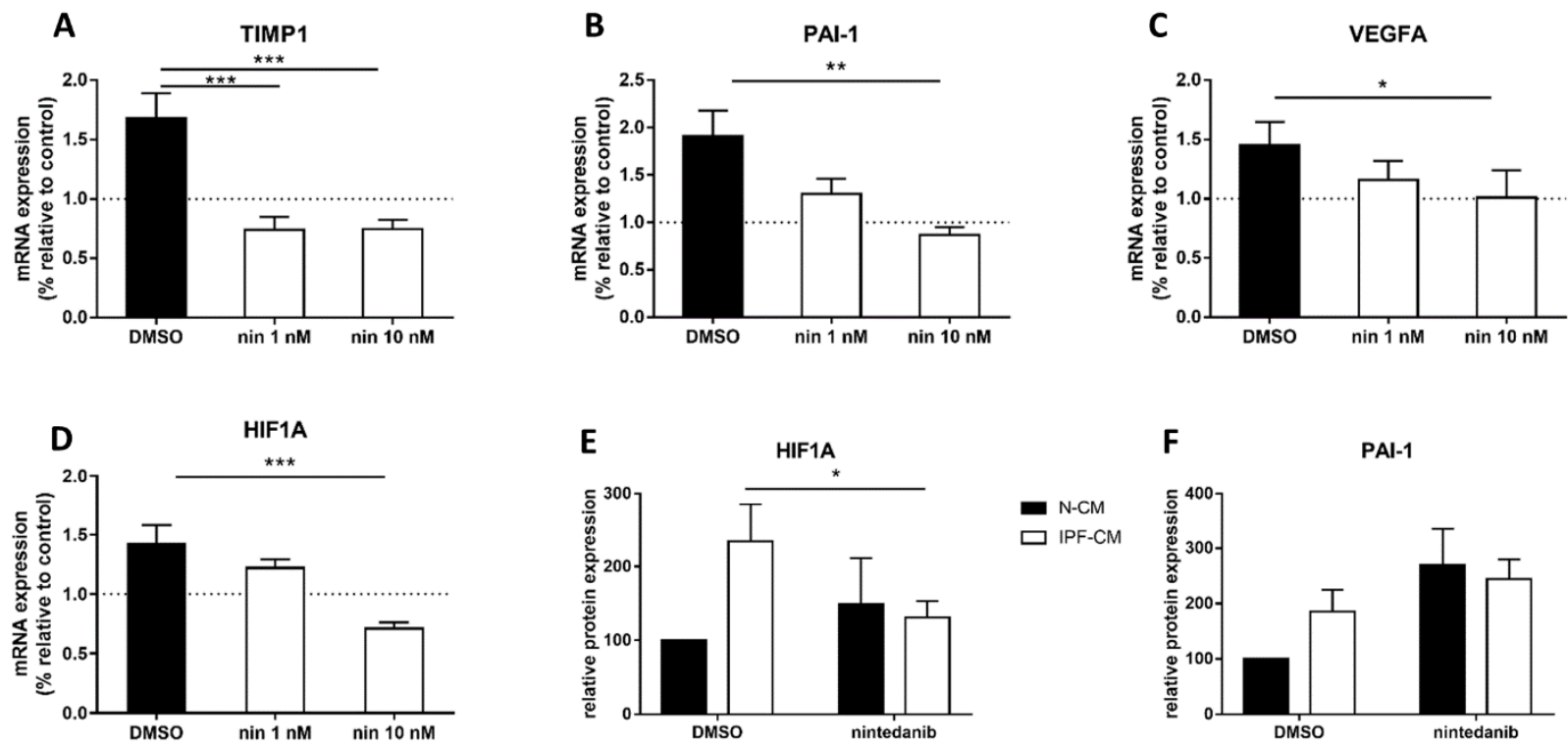

E

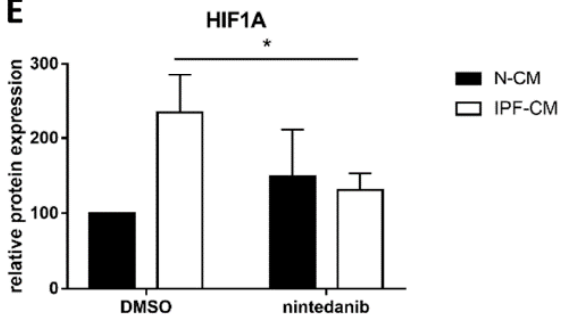

$\mathbf{F}$

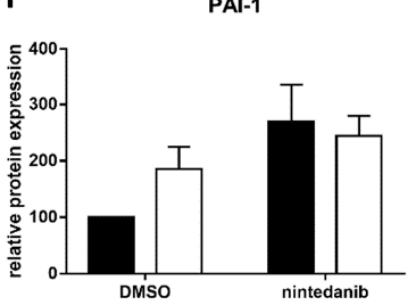

+ nintedanib

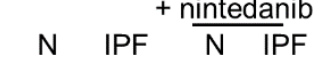

HIF1A

GAPDH
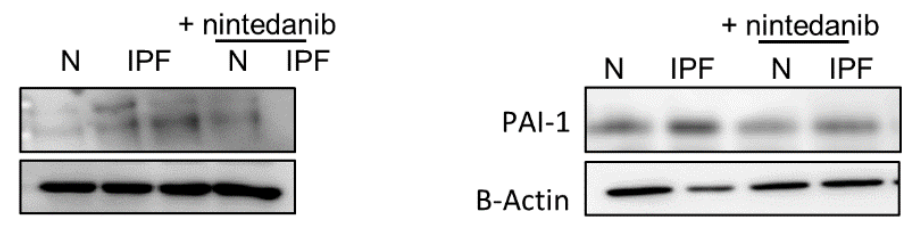

Figure 3. Nintedanib prevents the elevation of HIF1 $\alpha$-related targets. Normal human lung fibroblasts (N-HLF) were exposed to nintedanib (1-10 $\mathrm{nM}$ ) or DMSO for $60 \mathrm{~min}$ and the cultured on N/IPF-CM for $24 \mathrm{~h}$ additional without nintedanib or DMSO. Effects of the IPF-CM with/without nintedanib (nin) on TIMP1 $(n=6)$, PAI-1 $(n=6)$ and VEGFA $(n=6)$ and HIF1A $(n=6)$ mRNA levels and on HIF1 $\alpha(n=6)$ and PAI-1 $(n=6)$ protein levels were tested by qPCR $($ A-D) and Western Blot $(\mathbf{E}, \mathbf{F})$, respectively following $24 \mathrm{~h}$. Values are means \pm SEM. ${ }^{*} p<0.05,{ }^{* *} p<0.01$ and ${ }^{* * *} p<0.001$.

\subsection{Rat Bleomycin Model Shows Elevated Levels of HIF1 $\alpha$ within Fibrotic Tissue}

These findings were tested in an in-vivo model, which included rats exposed to bleomycin, followed by treatment with oral or inhaled vehicle/nintedanib [12]. Results showed that nintedanib was associated with lower numbers and smaller foci of fibrosis resulting in a lower median fibrosis score for animals in this group compared to inhaled vehicle treated controls. Here, we evaluated HIF1 $\alpha$, TIMP1 and PAI-1 in cell blocks from those experiments. In support of the above findings, HIF1 $\alpha$ expression was significantly elevated in rats exposed to bleomycin, whereas treatment with nintedanib significantly reduced these effects (ANOVA $p<0.0001$, Figure 4A). TIMP1 was weakly detected in a cytoplasmic staining in all samples and was also observed in mast cells within the fibrotic 
areas. PAI-1 was also increased in the bleomycin treated rats, yet to a lesser extent and was slightly reduced with nintedanib treatment. Interestingly, the high inhaled nintedanib dose $(0.375 \mathrm{mg} / \mathrm{kg})$ increased PAI-1 levels $(p<0.05$, Figure $4 \mathrm{~B})$, highlighting the importance of low dosing whenever possible.

A

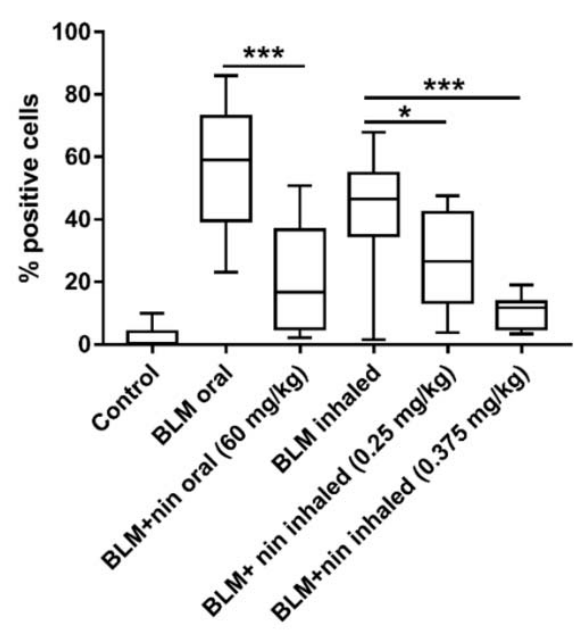

B

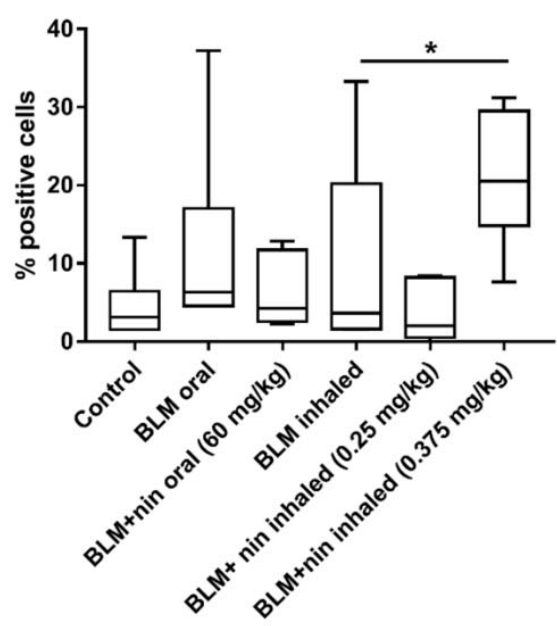

C

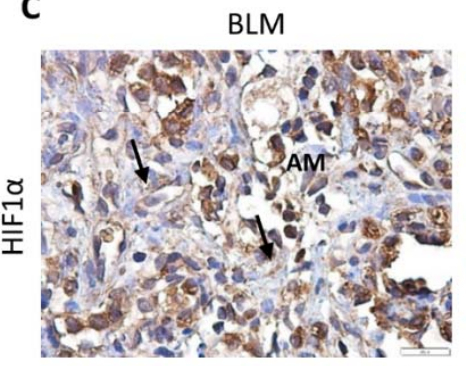

$\mathrm{BLM}+$ nintedanib (oral)

$\mathrm{BLM}+$ nintedanib (inhaled)
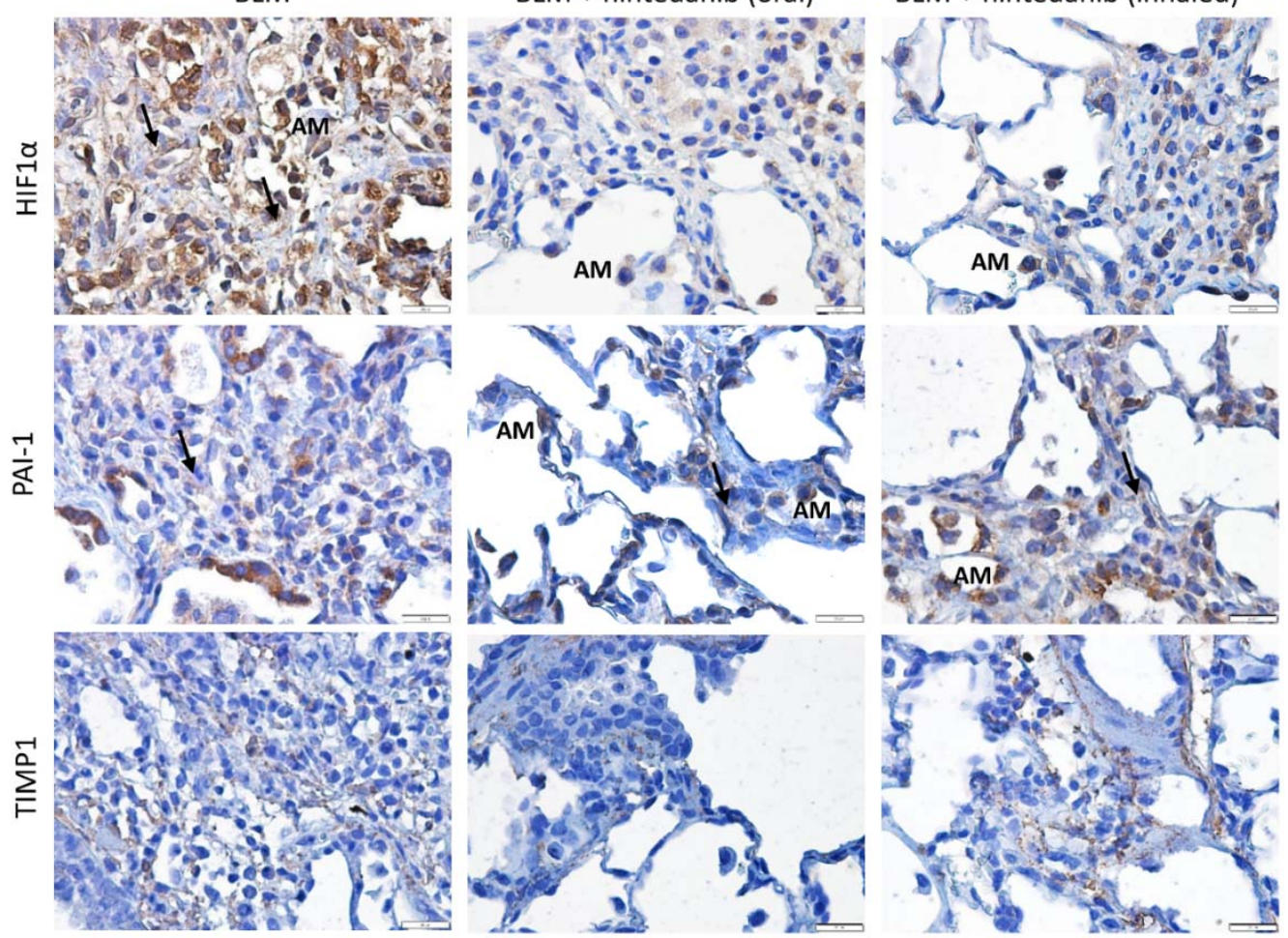

Figure 4. Rat Bleomycin model shows elevated levels of HIF1 $\alpha$ within fibrotic tissue. Formalin fixed paraffin embedded (FFPE) sections of rats treated with vehicle (control), bleomycin (BLM) with/without oral or inhaled nintedanib (nin) were stained for HIF1 $\alpha$ (A) $(n \geq 7)$, PAI-1 (B) $(n \geq 6)$ and TIMP1 by IHC and counter stained by hematoxylin. (C) Representative IHC for figures A,B and for TIMP1. ${ }^{*} p<0.05,{ }^{* * *} p<0.001$. AM-alveolar macrophages, arrows indicate fibroblasts. Magnification (X400). Arrows indicate staining of fibroblasts. 


\section{Discussion}

The pathogenesis of IPF is still incompletely understood, and is thought to involve recurrent injury to the alveolar epithelium inducing an aberrant wound healing response, characterized by activation and proliferation of fibroblasts and myofibroblasts [20]. With the aim to discover underlying mechanisms leading to myofibroblast differentiation, we used our established human IPF-CM in-vitro model, which is a platform that enables the investigation of fibroblast-ECM interactions. In the high-throughput RNA analysis, the HIF1 $\propto$ pathway was found to be the most significant.

HIF1 $\alpha$, a key transcriptional factor in response to this chronic hypoxia, is involved in many fibrotic diseases [21,22] and was shown to regulate the expression of over 200 genes in an oxygen-dependent and independent manner [23,24]. The presence of hypoxia is an established hallmark of chronic tissue injury and fibrosis [25,26]. McDonough et al. [27], analyzed microRNAs and target genes from different IPF stages. Their analysis showed 20 downregulated genes, all regulated by miR-21, which is well established in IPF [28]. Pathway enrichment for these 20 genes revealed that they were related to the HIF $1 \alpha$ signaling pathway ([KEGG]: 04066), as found in our analysis. HIF1 $\alpha$ was also shown as one of the major genes implicated in IPF in a Single-Cell Transcriptomic Analysis by Reyfman et al. [29].

HIF1 $\alpha$ was shown to promote the fibrotic phenotype of alternatively activated macrophages through increased cell differentiation profibrotic mediator production, such as IL-6 [30]. Data from pulmonary fibrosis mouse models [31] and IPF patients have revealed increased HIF1 $\alpha$ expression in alveolar epithelial cells at an early stage of pulmonary fibrosis [32]. However, HIF1A and HIF2A double knockout in ATII cells did not show protection against lung fibrosis induced by bleomycin in mice, suggesting that HIF signaling may not have important roles in ATII during the initial developmental steps of pulmonary fibrosis [33]. However, HIF1 $\alpha$ may act as an amplifier of the disease in fibroblasts, as suggested in our system [34].

Another interesting observation from our analysis was regarding the housekeeping genes. The notion of housekeeping genes has been in use in the literature for nearly 50 years. In particular, several genes have been widely used as internal controls in experimental expression studies, such as GAPDH, tubulins and 18S [35]. In our laboratory, we successfully used the GAPDH and $\alpha$ Tubulin, which showed no significant changes. Following the RNA-sequencing analysis, discovering that GAPDH is slightly elevated following IPF-CM exposure, we added HPRT1 and ACTB to our data, as they showed no change. Since performing sequencing for every experiment is not optional, this point should be carefully addressed in future experiments.

When analyzing IPF patient samples, HIF1 $\alpha$ was expressed at lower levels in fibroblastic foci, and at higher levels in collagenous "old fibrosis" areas. Supported by other works, HIF $1 \alpha$ has also been observed to be highly expressed in various cell types throughout the lung tissue, especially within the fibrotic areas [33]. In the rat bleomycin-treated tissue, HIF1 $\alpha$ was also significantly elevated. However, since the UIP pattern is specific to humans [27], similar analysis of fibroblastic foci vs. fibrotic tissue couldn't be performed.

TGF $\beta$ and hypoxia signaling appear interrelated wherein Smad3, the major TGF $\beta$ responsive transcription factor, can be up-regulated by hypoxia [36] and collagen expression by TGF- $\beta 1$ can be decreased by inhibiting HIF1 $\alpha$ [37]. Given these and other observations, Goodwin et al. suggested that fibroblast-specific HIF1 $\alpha$ signaling is a critical component of pulmonary fibrosis [26]. Similar to IPF-CM stimulating elevated $\alpha$ SMA in N-HLFs, they and others [38] showed that hypoxia can induce $\alpha$ SMA expression in N-HLFs. Interestingly, recent studies have shown that HIF1 $\alpha$ expression could be increased by additional nonhypoxic stimuli (e.g., PDGF, TNF $\alpha$, angiotensin II and a variety of growth factors) [34,39]. Thus, further research is needed to understand the trigger for HIF1 $\alpha$ overexpression.

Nevertheless, Goodwin et al. suggested that fibroblast-specific HIF1 $\alpha$ signaling is a critical component of pulmonary fibrosis [26]. Similar to IPF-CM stimulating elevated $\alpha \mathrm{SMA}$ in N-HLFs, they and others [38] showed that hypoxia can induce $\alpha \mathrm{SMA}$ expression 
in N-HLFs. Interestingly, recent studies showed that HIF1 $\alpha$ expression could be increased by additional non-hypoxic stimuli (e.g., PDGF, TNF $\alpha$, angiotensin II and a variety of growth factors) $[34,39]$. Whatever the trigger, HIF1 $\alpha$ and its target genes (SERPINE1, VEGFA and TIMP1) were significantly overexpressed in the IPF-CM-exposed N-HLFs. As previously described by McMahon et al. [11], factors such as TGF $\beta 1$ stabilizing the HIF1 $\alpha$, may also be implicated in the pro-fibrotic phenotype induced by IPF-CM. Our previous work showed that the TGF $\beta$ pathway can be activated by the IPF-CM [7].

Preclinical and clinical studies show that the fibrotic process correlates with the expression of HIF1 $\alpha$ target genes, such as TIMP1 and PAI-1 [32,40]. Among the numerous genes whose expression is regulated by TGF $\beta 1$ and HIF1 $\alpha$, PAI-1 has been shown to play a key role in pulmonary fibrosis development $[39,41]$. Moreover, bleomycin-induced fibrosis was shown to be more severe in transgenic mice overexpressing PAI-1, whereas PAI-1 knockout mice were protected from fibrosis. Ueno et al. showed that PAI-1 expression was highest on day 7 following bleomycin exposure and reduced thereafter [39]. Since our analysis was performed on day 28 , this may explain the relatively low levels detected. Interestingly, it was also shown that TGF $\beta 1$ exposure increased PAI-1 and HIF1 $\alpha$ protein, but not HIF1A mRNA levels [39]. These findings support our results, which suggest the HIF1 $\alpha$ overexpression is probably controlled at the post-transcriptional level.

VEGFA, another HIF1 $\alpha$ target was also elevated following HLF exposure to the IPF$\mathrm{CM}$, as well as in tissue lysates of patients with IPF. These findings support other works proposing that VEGFA might facilitate fibrogenesis [42,43]. However, as recently reviewed by Barratt et al. [19], it was stated that the relationship of VEGFA expression in IPF remains controversial and appears to differ according to the compartment sampled. Therefore, it remains unclear whether the upregulation in VEGFA is a protective mechanism or an inducer of fibrosis [44]. In the IPF-CM system, VEGFA mRNA expression was significantly blocked by nintedanib, which is known to inhibit the VEGF receptor [8,9]. Since VEGFA is a secreted factor, HLF protein expressions were not evaluated following culture on the IPF-CM. Additionally, since BALF or serum samples were not available, VEGFA levels were not further analyzed in the rat model.

In the last part of this work, the in-vivo impact of both oral and inhaled nintedanib on bleomycin-induced HIF1 $\alpha$ expression was studied. Similar to the histopathologic findings, both routes of nintedanib administration inhibited HIF1 $\alpha$ to a similar extent, with the inhaled high dose being most effective. However, PAI-1 differed where the higher inhaled dose showed elevated levels. One explanation could be that the high dose inhibited the progression of disease, and thus the high amounts represent a slower timeline of disease. To test this theory, earlier sections (from days 7-14) are needed that might show reduced PAI-1 expression with the high doses. Alternatively, the higher dose may have initiated additional signaling pathways, such as signal transducer and activator of transcription 3 (STAT3). STAT3 was previously shown to non-canonically activate Smad3 and therefore, possibly, PAI-1. In our recent work we showed that nintedanib can elevate pSTAT3 levels in HLFs [45].

TIMP1 was specifically observed in mast cells within the fibrotic areas. These findings correlate to a recent study by Overed-Sayer et al. [20] that showed induction of mast cells in IPF and could explain the lack of positive staining in the HLFs. Moreover, this group showed that nintedanib inhibits mast cell survival and activation and thus provides a novel additional mechanism by which this drug may exert anti-fibrotic effects in patients with IPF.

Stabilized HIF1 $\alpha$ under hypoxic conditions can trans-activate target genes involved in metabolic reprogramming into anaerobic glycolysis [46]. In fact, it was recently suggested that glycolytic metabolism may be the next focus point in pulmonary fibrotic progression. Zhao et al. recently showed that fibroblast catabolism of ECM could limit fibrotic progression [47]. Furthermore, there is growing literature examining the potential of targeting metabolism to treat "non-metabolic" conditions, such as fibrosis [48]. Accordingly, high lactic acid levels, indicative of up-regulated glycolysis, have been observed in patients 
with IPF, and were associated with augmented myofibroblast differentiation [49]. These results suggest that glycolytic reprograming is an important contributor to myofibroblast activation and differentiation, thereby promoting pulmonary fibrosis progression. Notably, the second most upregulated pathway in our RNA-sequencing analysis was "Glycolysis/Gluconeogenesis", including the SLC2A1 (GLUT1) gene, which was previously implicated in fibrosis progression and also known to be induced by TGF $\beta$ [50]. This direction was not explored in the current work, but highlights the complexity of events leading to fibrosis.

In conclusion, once formed, IPF-ECM sets up a pro-fibrotic feedback loop that is capable of sustaining progressive fibrosis. This loop was shown to include the HIF $1 \alpha$ signaling pathway. To our knowledge, we are the first to show the inhibition of this pathway by nintedanib. Although the complete mechanism was not fully elucidated, nintedanib was shown to reduce HIF1 $\alpha$ levels and to block the pro-fibrotic process propagated by the ECM.

\section{Materials and Methods}

\subsection{Primary HLF Culture}

Primary HLFs were isolated from IPF (histologically confirmed) tissues and control samples (histologically normal lung distant from the resected tumor) at the time of biopsy, as described [51]. Following extraction, HLFs were cultured in Dulbecco's Modified Eagle's Medium (DMEM) supplemented with 20\% FCS, L-glutamine $(2 \mathrm{mM})$ with antibiotics (Biological Industries, Beit-Haemek, Israel) and maintained in $5 \% \mathrm{CO}_{2}$ at $37^{\circ} \mathrm{C}$.

\subsection{IPF-CM Model}

Experiments were performed as previously described [7]. Briefly, IPF/N-HLFs were cultured on Matrigel (BD biosciences (by Corning), Glendale, AZ, USA). Following $48 \mathrm{~h}$, cells were removed by $\mathrm{NH}_{4} \mathrm{OH}$ and $\mathrm{N}-\mathrm{HLFs}$ were added for further culture. Nintedanib (1-100 nM, Avalyn Pharma, Seattle, WA, USA) was diluted in DMSO and added an hour prior to the addition of N-HFs to the culture.

\subsection{Western Blot}

Western blot was performed as previously described [51]. Antibodies are listed in Supplementary Table S1. Bound antibodies were visualized using Goat peroxidase-conjugated secondary antibodies (Supplementary Table S1, doi:10.6084/m9.figshare.13475100) followed by enhanced chemiluminescence detection (Millipore, Temecula, CA, USA). LAS3000 (Fujifilm, Japan) was used to quantify protein expressions.

\subsection{Real Time Quantitative PCR ( $q P C R)$}

RNA was extracted by RNeasy kit (Qiagen, Germany) and converted to cDNA using GeneAmp (Applied Biosystems, Foster City, CA, USA). Reactions were performed with SYBR Green (Applied Biosystems, Carlsbad, CA, USA). Primers are listed in Supplementary Table S2, doi:10.6084/m9.figshare.13475142).

\subsection{RNA-Sequencing}

RNA libraries were generated using CEL-Seq protocol, and sequenced on Illumina HiSeq2500, 15/50 paired-end run. Reads were trimmed using trim galore (cutadapt version 1.10) and mapped to the Human genome (ftp:/ / ftp.ensembl.org/pub/release-89/fasta/ homo_sapiens/dna/Homo_sapiens.GRCh38.dna.primary_assembly.fa.gz) accessed on 20 May 2020, using Tophat2 version 2.1.0 (uses Bowtie2 version 2.2.6, http:/ /www.ncbi. nlm.nih.gov / pubmed/19289445?dopt=Abstract, accessed on 20 May 2020), with up to 2 mismatches allowed per read. The minimum and maximum intron sizes were set to 70 and 500,000 , respectively, and an annotation file was provided to the mapper. Only uniquely mapped reads were counted to genes, using 'HTSeq-count' package version 0.6.1 with 'union' mode (http:/ / www.ncbi.nlm.nih.gov/pubmed/25260700?dopt=Abstract), accessed 
on 20 May 2020. Normalization and differential expression analyses were conducted using DESeq2 R version 1.18.1 package.

\subsection{Rat Bleomycin Model}

The study consisted of 7 treatment groups with 10 animals per group as previously described [12]. Bleomycin $(1 \mathrm{mg} / \mathrm{kg}$ in $100 \mu \mathrm{L})$ or vehicle was administered to animals on four occasions by oropharyngeal aspiration during Week 1 to induce lung fibrosis (Days 1 , 2, 3 and 6). Treatments were started on Day 8 after a one-day bleomycin recovery period. Oral nintedanib was administered twice daily (BID) by oral gavage at $60 \mathrm{mg} / \mathrm{kg}$. Inhaled nintedanib was administered once daily (QD) by oropharyngeal aspiration at 0.25 and $0.375 \mathrm{mg} / \mathrm{kg}$. Body weights were recorded daily. On Day 28, animals were euthanized, and terminal lung and body weights were obtained. The right lung from each animal was inflated and then immersion fixed with $10 \%$ neutral buffered formalin and embedded in paraffin. Three sections for the right caudal lobe were cut for the immunohistochemistry analysis.

\subsection{Immunohistochemistry (IHC)}

Sections prepared from patients with IPF and from the rat lungs were deparaffinized in xylene and alcohol, rinsed in PBS and immersed in citrate buffer ( $\mathrm{pH} 6$ ). Slides were incubated overnight at $4{ }^{\circ} \mathrm{C}$ with primary antibodies (Supplementary Table S1), and developed using ImmPACT DAB (Vector laboratories, Burlingame, CA, USA) according to manufacturers' instructions. Use of isotype-matched control eliminated non-specific staining. Expression levels were measured using Qupath [52]. Sections were counterstained with hematoxylin (Sigma, St. Louis, MO, USA). Fibroblastic foci, established fibrosis and non-fibrotic areas of the IPF lung sections were annotated by a registered senior pathologist.

\subsection{Statistical Analysis}

Analysis was done using GraphPad Prism (GraphPad Software, San Diego, CA, USA, www.graphpad.com, accessed on 20 May 2020) and by SPSS (IBM, Armonk, NY, USA). ANOVA was performed to compare differences between multiple cohorts. Student's $t$-tests were employed to analyze differences between two groups. An effect was considered significant for $p<0.05$.

\subsection{Ethics Statement}

The HLF study was approved by the Ethics Committee of the Meir Medical Center (MMC-016-16, issued on 30 July 2016). Informed consent was obtained from all patients. All animal experiments were performed in accordance with the UK Animals (Scientific Procedures) Act, 1986, under the authority and controls of a project license held at the Charles River Edinburgh facility.

Supplementary Materials: The following are available online at https: / www.mdpi.com/1422-0067/ 22/7/3331/s1, Table S1: doi:10.6084/m9.figshare.13475100, Table S2: doi:10.6084/m9.figshare.13475142.

Author Contributions: Conceptualization, M.R.K. and D.S.; data curation, B.B.-W., M.M. and A.K.; formal analysis, G.E.S., M.M., A.K., E.E. and D.S.; funding acquisition, M.S.; investigation, G.E.S. and B.B.-W.; methodology, G.E.S., M.M., A.K., M.S., E.E., B.P. and M.R.K.; project administration, B.B.-W., M.M., M.S. and B.P.; resources, M.S., B.P., M.R.K. and D.S.; validation, A.K., M.S. and E.E.; writing—original draft, G.E.S., B.B.-W., E.E. and B.P.; writing—review and editing, G.E.S., M.M., A.K., M.S., M.R.K. and D.S. All authors have read and agreed to the published version of the manuscript.

Funding: In-vivo studies were funded by Avalyn Pharma. No additional funding was received for this project.

Institutional Review Board Statement: The HLF study was approved by the Ethics Committee of the Meir Medical Center (MMC-016-16, issued on 30 July 2016). All animal experiments were performed in accordance with the UK Animals (Scientific Procedures) Act, 1986, under the authority and controls of a project license held at the Charles River Edinburgh facility. 
Informed Consent Statement: Informed consent was obtained from all subjects involved in the study.

Data Availability Statement: Any data can be supplemented on demand.

Acknowledgments: We thank Tatiana Epstein for the English editing and to Avivit, Amany and Inna from the pathology department for their help with slide preparation. This work was part of Becky Bardensteins' MSc thesis in Tel-Aviv University.

Conflicts of Interest: All authors declare no conflict of interest and have consented for publication. Mark Surber is employed at Avalyn Pharma. The funders participated in the design of the study, yet had no role in the collection, analyses, or interpretation of data; in the writing of the manuscript, or in the decision to publish the results.

\section{References}

1. Gribbin, J.; Hubbard, R.B.; Le Jeune, I.; Smith, C.J.; West, J.; Tata, L.J. Incidence and mortality of idiopathic pulmonary fibrosis and sarcoidosis in the UK. Thorax 2006, 61, 980-985. [CrossRef]

2. Fielding, C.A.; Jones, G.W.; McLoughlin, R.M.; McLeod, L.; Hammond, V.J.; Uceda, J.; Williams, A.S.; Lambie, M.; Foster, T.L.; Liao, C.T.; et al. Interleukin-6 signaling drives fibrosis in unresolved inflammation. Immunity 2014, 40, 40-50. [CrossRef] [PubMed]

3. Sgalla, G.; Iovene, B.; Calvello, M.; Ori, M.; Varone, F.; Richeldi, L. Idiopathic pulmonary fibrosis: Pathogenesis and management. Respir. Res. 2018, 19, 32. [CrossRef]

4. Richter, K.; Kietzmann, T. Reactive oxygen species and fibrosis: Further evidence of a significant liaison. Cell Tissue Res. 2016, 365, 591-605. [CrossRef] [PubMed]

5. Wynn, T.A. Integrating mechanisms of pulmonary fibrosis. J. Exp. Med. 2011, 208, 1339-1350. [CrossRef]

6. Herrera, J.; Henke, C.A.; Bitterman, P.B. Extracellular matrix as a driver of progressive fibrosis. J. Clin. Investig. 2018, 128, 45-53. [CrossRef]

7. Epstein Shochet, G.; Wollin, L.; Shitrit, D. Fibroblast-matrix interplay: Nintedanib and pirfenidone modulate the effect of IPF fibroblast-conditioned matrix on normal fibroblast phenotype. Respirology 2018, 23, 756-763. [CrossRef]

8. Hilberg, F.; Roth, G.J.; Krssak, M.; Kautschitsch, S.; Sommergruber, W.; Tontsch-Grunt, U.; Garin-Chesa, P.; Bader, G.; Zoephel, A.; Quant, J.; et al. BIBF 1120: Triple angiokinase inhibitor with sustained receptor blockade and good antitumor efficacy. Cancer Res. 2008, 68, 4774-4782. [CrossRef]

9. Wollin, L.; Maillet, I.; Quesniaux, V.; Holweg, A.; Ryffel, B. Antifibrotic and anti-inflammatory activity of the tyrosine kinase inhibitor nintedanib in experimental models of lung fibrosis. J. Pharmacol. Exp. Ther. 2014, 349, 209-220. [CrossRef] [PubMed]

10. Rangarajan, S.; Kurundkar, A.; Kurundkar, D.; Bernard, K.; Sanders, Y.Y.; Ding, Q.; Antony, V.B.; Zhang, J.; Zmijewski, J.; Thannickal, V.J. Novel Mechanisms for the Antifibrotic Action of Nintedanib. Am. J. Respir. Cell Mol. Biol. 2016, 54, 51-59. [CrossRef]

11. McMahon, S.; Charbonneau, M.; Grandmont, S.; Richard, D.E.; Dubois, C.M. Transforming growth factor beta1 induces hypoxiainducible factor-1 stabilization through selective inhibition of PHD2 expression. J. Biol. Chem. 2006, 281, 24171-24181. [CrossRef] [PubMed]

12. Surber, M.W.; Beck, S.; Pham, S.; Marsden, A.T.; Gandi, S.K.; Baily, J.; McElroy, M.C. Inhaled nintedanib is well-tolerated and delivers key pharmacokinetic parameters required to treat bleomycin-induced pulmonary fibrosis. Pulm. Pharmacol. Ther. 2020, 63, 101938. [CrossRef]

13. Epstein-Shochet, G.; Pham, S.; Beck, S.; Naiel, S.; Mekhael, O.; Revill, S.; Hayat, A.; Vierhout, M.; Bardestein-Wald, B.; Shitrit, D.; et al. Inhalation: A means to explore and optimize nintedanib's pharmacokinetic/pharmacodynamic relationship. Pulm. Pharmacol. Ther. 2020. [CrossRef]

14. Wang, Y.; Yella, J.K.; Ghandikota, S.; Cherukuri, T.C.; Ediga, H.H.; Madala, S.K.; Jegga, A.G. Pan-transcriptome-based Candidate Therapeutic Discovery for Idiopathic Pulmonary Fibrosis. bioRxiv 2020, 824367. [CrossRef] [PubMed]

15. Liao, Y.; Wang, J.; Jaehnig, E.J.; Shi, Z.; Zhang, B. WebGestalt 2019: Gene set analysis toolkit with revamped UIs and APIs. Nucleic Acids Res. 2019, 47, W199-W205. [CrossRef] [PubMed]

16. Huang da, W.; Sherman, B.T.; Lempicki, R.A. Systematic and integrative analysis of large gene lists using DAVID bioinformatics resources. Nat. Protoc. 2009, 4, 44-57. [CrossRef] [PubMed]

17. Ghosh, A.K.; Vaughan, D.E. PAI-1 in tissue fibrosis. J. Cell Physiol. 2012, 227, 493-507. [CrossRef]

18. Yoshiji, H.; Kuriyama, S.; Miyamoto, Y.; Thorgeirsson, U.P.; Gomez, D.E.; Kawata, M.; Yoshii, J.; Ikenaka, Y.; Noguchi, R.; Tsujinoue, H.; et al. Tissue inhibitor of metalloproteinases-1 promotes liver fibrosis development in a transgenic mouse model. Hepatology 2000, 32, 1248-1254. [CrossRef] [PubMed]

19. Barratt, S.L.; Flower, V.A.; Pauling, J.D.; Millar, A.B. VEGF (Vascular Endothelial Growth Factor) and Fibrotic Lung Disease. Int. J. Mol. Sci. 2018, 19, 1269. [CrossRef]

20. Overed-Sayer, C.; Miranda, E.; Dunmore, R.; Liarte Marin, E.; Beloki, L.; Rassl, D.; Parfrey, H.; Carruthers, A.; Chahboub, A.; Koch, S.; et al. Inhibition of mast cells: A novel mechanism by which nintedanib may elicit anti-fibrotic effects. Thorax 2020, 75, 754-763. [CrossRef] 
21. Xiong, A.; Liu, Y. Targeting Hypoxia Inducible Factors-1alpha As a Novel Therapy in Fibrosis. Front. Pharmacol. 2017, 8, 326. [CrossRef] [PubMed]

22. Zhan, L.; Huang, C.; Meng, X.M.; Song, Y.; Wu, X.Q.; Yang, Y.; Li, J. Hypoxia-inducible factor-1alpha in hepatic fibrosis: A promising therapeutic target. Biochimie 2015, 108, 1-7. [CrossRef] [PubMed]

23. Cummins, E.P.; Keogh, C.E. Respiratory gases and the regulation of transcription. Exp. Physiol. 2016, 101, 986-1002. [CrossRef] [PubMed]

24. Semenza, G.L. Oxygen-regulated transcription factors and their role in pulmonary disease. Respir. Res. 2000, 1, 159-162. [CrossRef] [PubMed]

25. Lokmic, Z.; Musyoka, J.; Hewitson, T.D.; Darby, I.A. Hypoxia and hypoxia signaling in tissue repair and fibrosis. Int. Rev. Cell Mol. Biol. 2012, 296, 139-185. [CrossRef]

26. Goodwin, J.; Choi, H.; Hsieh, M.H.; Neugent, M.L.; Ahn, J.M.; Hayenga, H.N.; Singh, P.K.; Shackelford, D.B.; Lee, I.K.; Shulaev, V.; et al. Targeting Hypoxia-Inducible Factor-1alpha/Pyruvate Dehydrogenase Kinase 1 Axis by Dichloroacetate Suppresses Bleomycin-induced Pulmonary Fibrosis. Am. J. Respir. Cell Mol. Biol. 2018, 58, 216-231. [CrossRef]

27. McDonough, J.E.; Ahangari, F.; Li, Q.; Jain, S.; Verleden, S.E.; Herazo-Maya, J.; Vukmirovic, M.; DeIuliis, G.; Tzouvelekis, A.; Tanabe, N.; et al. Transcriptional regulatory model of fibrosis progression in the human lung. JCI Insight 2019, 4. [CrossRef] [PubMed]

28. Liu, G.; Friggeri, A.; Yang, Y.; Milosevic, J.; Ding, Q.; Thannickal, V.J.; Kaminski, N.; Abraham, E. miR-21 mediates fibrogenic activation of pulmonary fibroblasts and lung fibrosis. J. Exp. Med. 2010, 207, 1589-1597. [CrossRef]

29. Reyfman, P.A.; Walter, J.M.; Joshi, N.; Anekalla, K.R.; McQuattie-Pimentel, A.C.; Chiu, S.; Fernandez, R.; Akbarpour, M.; Chen, C.I.; Ren, Z.; et al. Single-Cell Transcriptomic Analysis of Human Lung Provides Insights into the Pathobiology of Pulmonary Fibrosis. Am. J. Respir Crit. Care Med. 2019, 199, 1517-1536. [CrossRef] [PubMed]

30. Philip, K.; Mills, T.W.; Davies, J.; Chen, N.Y.; Karmouty-Quintana, H.; Luo, F.; Molina, J.G.; Amione-Guerra, J.; Sinha, N.; Guha, A.; et al. HIF1A up-regulates the ADORA2B receptor on alternatively activated macrophages and contributes to pulmonary fibrosis. FASEB J. 2017, 31, 4745-4758. [CrossRef] [PubMed]

31. Delbrel, E.; Soumare, A.; Naguez, A.; Label, R.; Bernard, O.; Bruhat, A.; Fafournoux, P.; Tremblais, G.; Marchant, D.; Gille, T.; et al. HIF-1alpha triggers ER stress and CHOP-mediated apoptosis in alveolar epithelial cells, a key event in pulmonary fibrosis. Sci. Rep. 2018, 8, 17939. [CrossRef]

32. Tzouvelekis, A.; Harokopos, V.; Paparountas, T.; Oikonomou, N.; Chatziioannou, A.; Vilaras, G.; Tsiambas, E.; Karameris, A.; Bouros, D.; Aidinis, V. Comparative expression profiling in pulmonary fibrosis suggests a role of hypoxia-inducible factor-1alpha in disease pathogenesis. Am. J. Respir. Crit. Care Med. 2007, 176, 1108-1119. [CrossRef]

33. Burman, A.; Kropski, J.A.; Calvi, C.L.; Serezani, A.P.; Pascoalino, B.D.; Han, W.; Sherrill, T.; Gleaves, L.; Lawson, W.E.; Young, L.R.; et al. Localized hypoxia links ER stress to lung fibrosis through induction of C/EBP homologous protein. JCI Insight $2018,3$. [CrossRef] [PubMed]

34. Lee, J.W.; Ko, J.; Ju, C.; Eltzschig, H.K. Hypoxia signaling in human diseases and therapeutic targets. Exp. Mol. Med. 2019, 51, 1-13. [CrossRef]

35. Eisenberg, E.; Levanon, E.Y. Human housekeeping genes, revisited. Trends Genet. 2013, 29, 569-574. [CrossRef]

36. Zhang, H.; Akman, H.O.; Smith, E.L.; Zhao, J.; Murphy-Ullrich, J.E.; Batuman, O.A. Cellular response to hypoxia involves signaling via Smad proteins. Blood 2003, 101, 2253-2260. [CrossRef] [PubMed]

37. Basu, R.K.; Hubchak, S.; Hayashida, T.; Runyan, C.E.; Schumacker, P.T.; Schnaper, H.W. Interdependence of HIF-1alpha and TGF-beta/Smad3 signaling in normoxic and hypoxic renal epithelial cell collagen expression. Am. J. Physiol. Renal. Physiol. 2011, 300, F898-F905. [CrossRef]

38. Aquino-Galvez, A.; Gonzalez-Avila, G.; Jimenez-Sanchez, L.L.; Maldonado-Martinez, H.A.; Cisneros, J.; Toscano-Marquez, F.; Castillejos-Lopez, M.; Torres-Espindola, L.M.; Velazquez-Cruz, R.; Rodriguez, V.H.O.; et al. Dysregulated expression of hypoxia-inducible factors augments myofibroblasts differentiation in idiopathic pulmonary fibrosis. Respir. Res. 2019, 20, 130. [CrossRef] [PubMed]

39. Ueno, M.; Maeno, T.; Nomura, M.; Aoyagi-Ikeda, K.; Matsui, H.; Hara, K.; Tanaka, T.; Iso, T.; Suga, T.; Kurabayashi, M. Hypoxiainducible factor-1alpha mediates TGF-beta-induced PAI-1 production in alveolar macrophages in pulmonary fibrosis. Am. J. Physiol. Lung Cell Mol. Physiol. 2011, 300, L740-L752. [CrossRef]

40. Kaminski, N.; Rosas, I.O. Gene expression profiling as a window into idiopathic pulmonary fibrosis pathogenesis: Can we identify the right target genes? Proc. Am. Thorac. Soc. 2006, 3, 339-344. [CrossRef]

41. Olman, M.A.; Mackman, N.; Gladson, C.L.; Moser, K.M.; Loskutoff, D.J. Changes in procoagulant and fibrinolytic gene expression during bleomycin-induced lung injury in the mouse. J. Clin. Investig. 1995, 96, 1621-1630. [CrossRef]

42. Hamada, N.; Kuwano, K.; Yamada, M.; Hagimoto, N.; Hiasa, K.; Egashira, K.; Nakashima, N.; Maeyama, T.; Yoshimi, M.; Nakanishi, Y. Anti-vascular endothelial growth factor gene therapy attenuates lung injury and fibrosis in mice. J. Immunol. 2005, 175, 1224-1231. [CrossRef]

43. Fehrenbach, H.; Kasper, M.; Haase, M.; Schuh, D.; Muller, M. Differential immunolocalization of VEGF in rat and human adult lung, and in experimental rat lung fibrosis: Light, fluorescence, and electron microscopy. Anat. Rec. 1999, 254, 61-73. [CrossRef]

44. Tzouvelekis, A.; Anevlavis, S.; Bouros, D. Angiogenesis in interstitial lung diseases: A pathogenetic hallmark or a bystander? Respir. Res. 2006, 7, 82. [CrossRef] [PubMed] 
45. Shochet, G.E.; Pomerantz, A.; Shitrit, D.; Bardenstein-Wald, B.; Ask, K.; Surber, M.; Rabinowicz, N.; Levy, Y.; Benchetrit, S.; Edelstein, E.; et al. Galectin-3 levels are elevated following nintedanib treatment. Ther. Adv. Chronic Dis. 2020, 11, 2040622320968412. [CrossRef]

46. Semenza, G.L. Regulation of metabolism by hypoxia-inducible factor 1. Cold Spring Harb Symp Quant. Biol 2011, 76, 347-353. [CrossRef]

47. Zhao, X.; Psarianos, P.; Ghoraie, L.S.; Yip, K.; Goldstein, D.; Gilbert, R.; Witterick, I.; Pang, H.; Hussain, A.; Lee, J.H.; et al. Metabolic regulation of dermal fibroblasts contributes to skin extracellular matrix homeostasis and fibrosis. Nat. Metab. 2019, 1, 147-157. [CrossRef] [PubMed]

48. Rabinowitz, J.D.; Mutlu, G.M. A metabolic strategy to reverse fibrosis? Nat. Metab. 2019, 1, 12-13. [CrossRef]

49. Kottmann, R.M.; Kulkarni, A.A.; Smolnycki, K.A.; Lyda, E.; Dahanayake, T.; Salibi, R.; Honnons, S.; Jones, C.; Isern, N.G.; Hu, J.Z.; et al. Lactic acid is elevated in idiopathic pulmonary fibrosis and induces myofibroblast differentiation via $\mathrm{pH}$-dependent activation of transforming growth factor-beta. Am. J. Respir. Crit. Care Med. 2012, 186, 740-751. [CrossRef]

50. Andrianifahanana, M.; Hernandez, D.M.; Yin, X.; Kang, J.H.; Jung, M.Y.; Wang, Y.; Yi, E.S.; Roden, A.C.; Limper, A.H.; Leof, E.B. Profibrotic up-regulation of glucose transporter 1 by TGF-beta involves activation of MEK and mammalian target of rapamycin complex 2 pathways. FASEB J. 2016, 30, 3733-3744. [CrossRef]

51. Epstein Shochet, G.; Brook, E.; Israeli-Shani, L.; Edelstein, E.; Shitrit, D. Fibroblast paracrine TNF-alpha signaling elevates integrin A5 expression in idiopathic pulmonary fibrosis (IPF). Respir. Res. 2017, 18, 122. [CrossRef] [PubMed]

52. Bankhead, P.; Loughrey, M.B.; Fernandez, J.A.; Dombrowski, Y.; McArt, D.G.; Dunne, P.D.; McQuaid, S.; Gray, R.T.; Murray, L.J.; Coleman, H.G.; et al. QuPath: Open source software for digital pathology image analysis. Sci. Rep. 2017, 7, 16878. [CrossRef] [PubMed] 